\title{
Preclinical assessment of a novel small molecule inhibitor of indoleamine 2,3-dioxygenase 1 (IDO1)
}

\author{
Gregory Driessens ${ }^{1}$, Stefano Crosignani ${ }^{1}$, Michel Detheux ${ }^{1}$ Benoît Van den Eynde ${ }^{2}$, Sandra Cauwenberghs ${ }^{*}$ \\ From Society for Immunotherapy of Cancer 29th Annual Meeting \\ National Harbor, MD, USA. 6-9 November 2014
}

\begin{abstract}
Introduction
iTeos leverages the science of the LICR to target the metabolism of the tumor microenvironment and develops small-molecule inhibitors. Tryptophan catabolism can suppress the anti-tumor immune response through expression of the rate-limiting enzyme IDO1. Local tryptophan reduction and metabolite production by the kynurenine pathway are associated with anergy/apoptosis of tumor-infiltrating lymphocytes. Two IDO1 inhibitors (Incyte INCB24360/NewLink NLG919) are currently tested in clinical trials for treatment of relapsed/refractory solid tumors but exhibit therapeutic limitations.
\end{abstract}

\section{Methods/results}

A primary HTS (176,000 compounds) led to the discovery of several confirmed hits. Medicinal chemistry optimization resulted in an original lead with $\mathrm{nM}$ potency in a relevant human blood assay, comparable to Incyte $\mathrm{IDO}_{\mathrm{i}}\left(\mathrm{IC}_{50} 890 \pm 220 \mathrm{nM}\right.$ [iTeos IDO1 $1_{\mathrm{i}}$ ] versus $970 \pm$ $100 \mathrm{nM}$ [Incyte IDO1 ${ }_{\mathrm{i}}$ ] [ $\mathrm{N}=3$ donors; Kyn ELISA]). For rodents \& monkey, iTeos compound showed low clearance $(9.9 \& 1.9 \mathrm{~mL} / \mathrm{min} / \mathrm{kg}$ respectively), moderate-to-low protein binding $(48 \& 28 \% \mathrm{Fu}$ respectively), a short halflife in rodents but long in monkey $(1.1 \& 12 \mathrm{~h}$ respectively), good oral availability ( $>75 \%$ ), and no inhibition on five CYP450 isoforms). Good predictive values for human PK were obtained through allometric scaling. Quantification of the tryptophan metabolite kynurenine showed a significant decrease in mouse plasma after oral administration of the IDO $1_{\mathrm{i}}$ in a dose response setting $\left(45.3 \pm 2.4\right.$ [iTeos $\mathrm{IDO}_{\mathrm{i}}$ ] and $48.1 \% \pm 3.0$ [Incyte IDO $1_{\mathrm{i}}$ ]; $2 \mathrm{~h}$ per os at $100 \mathrm{mg} / \mathrm{kg}$ compared to vehicle; $\mathrm{N}$ $=5$ independent experiments). In a time-course experiment, inhibition of kynurenine production lasted $8 \mathrm{~h}$ (iTeos IDO $1_{\mathrm{i}}$ ) compared to $16 \mathrm{~h}$ (Incyte IDO $1_{\mathrm{i}}$ ), consistent with their respective rodent half-life. A comparable PD effect was observed in mouse tumor lysates. Monkey PD is ongoing. Preliminary data obtained with the Pan02 mouse tumor model showed significant survival benefit of the iTeos IDO1 $1_{\mathrm{i}}$ in stand-alone and in combination with anti-CTLA4 treatment compared to vehicle or anti-CTLA4 in stand-alone respectively. 15 different human cancer types (60 samples each) were analyzed for IDO1 protein expression and allowed identification of several tumors with high expression.

\section{Conclusions}

iTeos' drug discovery efforts have delivered a selective IDO1 small-molecule inhibitor with novel scaffold suitable for in vitro/in vivo validation and with good predictive human PK (low clearance $\left(<10 \% \mathrm{Q}_{\mathrm{H}}\right.$ ), long half-life ( $>20 \mathrm{~h}$ ), high oral bioavailability).

\section{Authors' details}

${ }^{1}$ iTeos Therapeutics, Gosselies, Belgium. ${ }^{2}$ Ludwig Institute for Cancer Research (LICR, Brussels Branch) and de Duve Institute, Université catholique de Louvain (UCL), Brussels, Belgium.

Published: 6 November 2014

doi:10.1186/2051-1426-2-S3-P195

Cite this article as: Driessens et al:: Preclinical assessment of a novel small molecule inhibitor of indoleamine 2,3-dioxygenase 1 (IDO1). Journal for ImmunoTherapy of Cancer 2014 2(Suppl 3):P195.

${ }^{1}$ iTeos Therapeutics, Gosselies, Belgium

Full list of author information is available at the end of the article 